\title{
Molecular identification of the chitinase genes in Plasmodium relictum
}

\author{
Luz Garcia-Longoria ${ }^{1 *}$, Olof Hellgren² and Staffan Bensch²
}

\begin{abstract}
Background: Malaria parasites need to synthesize chitinase in order to go through the peritrophic membrane, which is created around the mosquito midgut, to complete its life cycle. In mammalian malaria species, the chitinase gene comprises either a large or a short copy. In the avian malaria parasites Plasmodium gallinaceum both copies are present, suggesting that a gene duplication in the ancestor to these extant species preceded the loss of either the long or the short copy in Plasmodium parasites of mammals. Plasmodium gallinaceum is not the most widespread and harmful parasite of birds. This study is the first to search for and identify the chitinase gene in one of the most prevalent avian malaria parasites, Plasmodium relictum.
\end{abstract}

Methods: Both copies of $P$. gallinaceum chitinase were used as reference sequences for primer design. Different sequences of Plasmodium spp. were used to build the phylogenetic tree of chitinase gene.

Results: The gene encoding for chitinase was identified in isolates of two mitochondrial lineages of $P$. relictum (SGS1 and GRW4). The chitinase found in these two lineages consists both of the long (PrCHT1) and the short (PrCHT2) copy. The genetic differences found in the long copy of the chitinase gene between SGS1 and GRW4 were higher than the difference observed for the cytochrome b gene.

Conclusion: The identification of both copies in P. relictum sheds light on the phylogenetic relationship of the chitinase gene in the genus Plasmodium. Due to its high variability, the chitinase gene could be used to study the genetic population structure in isolates from different host species and geographic regions.

Keywords: Avian malaria, Chitinase, Plasmodium relictum, SGS1, GRW4

\section{Background}

Malaria parasites have a complicated life cycle that requires several unique adaptive mechanisms that enable the parasite to successfully invade a variety of different tissues both in the vertebrate host and in the arthropod vector. Presumably as a protection against pathogens, arthropods develop a protective peritrophic membrane (PM) around their midgut after each blood meal which remains for 24 hours and then disappears [1]. The PM acts as a barrier blocking the penetration of parasites and not allowing them to spread to other organs [2]. Parasites in turn, have developed three different ways to overcome this barrier by (i) leaving the erythrocytes before the formation of the PM (as is the case Wuchereria infection) [3], (ii) persisting until the PM disappears (e.g. Leishmania) [4], or (iii) penetrating the PM (e.g.

\footnotetext{
*Correspondence: luzlongoria@unex.es

'Departamento de Biología Animal, Universidad de Extremadura, E-06071

Badajoz, Spain

Full list of author information is available at the end of the article
}

malaria parasites) [1]. The mechanism which allows malaria parasites to go through the PM of mosquitoes is well described [5-7]. These studies have shown that following the sexual process that takes place in the mosquito stomach, the ookinete has the ability to cross the PM by secreting a chitinase with characteristics of the family 18glycohydrolases that have catalytic and substrate-binding sites that breaks down this layer [8-10]. After crossing the PM, ookinetes finally transform into oocysts which after maturing (9-11 days [11]) releases the sporozoites that move to the salivary glands where they are ready for infecting a new host (e.g. birds). Therefore, chitinase secretion has an essential role in the completion of the life cycle of malaria parasites.

The mammalian Plasmodium parasite species have a single copy of the chitinase gene but with two different structures. In the human and primate malaria parasites, Plasmodium vivax and Plasmodium knowlesi and the 
rodent parasites (Plasmodium berghei, Plasmodium yoelii and Plasmodium chabaudi) the chitinase gene is longer and contains both a catalytic domain and a chitin-binding domain; in contrast, the shorter version present in Plasmodium falciparum and Plasmodium reichenowi lacks the chitin-binding domain [12]. Remarkably, the chicken parasite Plasmodium gallinaceum has functional copies of both the long (PgCHT1) and the short (PgCHT2) chitinase gene [12] suggesting that it is a common ancestor of the mammalian Plasmodium parasites that subsequently lost either the short or the long copy of the chitinase gene [13]. The phylogenetic relationships among Plasmodium parasites infecting mammals and birds have been intensively debated over the past decades. Some studies have found support for that $P$. falciparum is more closely related to bird parasites than to the other mammalian malaria parasites [13,14], whereas other studies support that the mammalian parasites are forming a monophyletic clade $[15,16]$. Because $P$. gallinaceum so far is the only bird malaria parasite investigated for its chitinase genes, it is too early to establish that the occurrence of both chitinase copies is representative for bird malaria parasites in general.

Plasmodium gallinaceum has been the primary model for studies related with chitinase function in avian malaria $[1,17]$. However, this species is not the most common malaria parasite in birds. In fact, species belonging to the genus Plasmodium show distinct differences in their distribution and prevalence [18]. The most widespread and harmful avian malaria species is Plasmodium relictum, found to infect more than 70 different bird species, whereas P. gallinaceum has been found to infect only 4 (MalAvi data base 2013-12-02 [19]). Plasmodium relictum is one of the most generalist malaria parasite in birds and has several mitochondrial cytochrome b lineages (e.g. SGS1, GRW4, GRW11, LZFUS01) that can be found in almost all continents (MalAvi data base 2013-12-02 [19]). Full understanding of the genetic mechanisms of the infection cycle could help to gain insights into why some parasites are specialist whereas others can infect a large number of different host species.

Despite the wide distribution and harmfulness of $P$. relictum, no study has tried to determine either if this species has the chitinase gene, nor the number of copies it possesses. Therefore, the objectives of this study were (1) to determine whether the two most widespread lineages of P. relictum (SGS1 and GRW4) have the gene encoding for chitinase, (2) if these lineages have both copies (CHT1 and CHT2) and (3) finally determine the genetic variability of chitinase genes between the lineages SGS1 and GRW4.

\section{Methods}

\section{Chitinase identification and sequencing}

Geneious 6.1. software primer design tool was used to create primers for amplification of overlapping partial regions within the catalytic domain of both copies of the P. gallinaceum chitinase genes (long: AF064079; short: AY842482). Each of the copies of the chitinase gene was first aligned to the sequences of all other available mammalian Plasmodium parasites in order to identify conserved regions. Figure 1 shows the position of the primers in both fragments of $P$. gallinaceum and the primer sequences are given in Table 1.

Two samples from previous experimental infections with $P$. relictum, the cytochrome b lineage SGS1 from crossbills [20] and GRW04 from great reed warblers [21], were used as DNA template. Total genomic DNA from the avian blood samples was extracted by standard ammonium acetate protocol [22]. All samples were screened for chitinase using a nested PCR method for chitinase genes with primers as in Table 1. For both steps, PCR reactions were set up in total volumes of $25 \mu \mathrm{l}$, containing $15.4 \mu \mathrm{l}$ of $\mathrm{ddH} 2 \mathrm{O}, 1.5 \mu \mathrm{l}$ of $\mathrm{MgCl} 2$ (25 mM), $2.5 \mu \mathrm{ldNTP}(10 \mathrm{mM}), 2.5 \mu \mathrm{l} \mathrm{10x}$ Buffer, $1 \mu \mathrm{l}$ of each primer $(10 \mu \mathrm{M}), 0.1$ of Taq polymerase and $1 \mu \mathrm{l}$ of each sample ( $25 \mathrm{ng} \mathrm{DNA} / \mu \mathrm{l})$. The PCR temperature profile was $95^{\circ} \mathrm{C}$ for $2 \mathrm{~min}$ followed by 25 or 35 cycles of $95^{\circ} \mathrm{C}$ for $30 \mathrm{sec}$, annealing temperature according to Table 1 for $30 \mathrm{sec}$ and $72^{\circ} \mathrm{C}$ for $30 \mathrm{sec}$ and terminated by a step of $72^{\circ} \mathrm{C}$ for $10 \mathrm{~min}$. For the SGS1 isolate we used an additional set of primers (PgCHT1_F3, PgCHT1_R3, PgCHT2_F3, PgCHT2R4) to amplify a region 3' to the fragment obtained with the nested protocol. Positive amplifications were precipitated and sequenced using a dye terminator cycling sequencing (big dye) kit and loaded on an ABI PRISM ${ }^{\mathrm{mi}} 3100$ sequencing robot (Applied Biosystems. Florida. USA).

\section{Phylogenetic analysis}

Sequences from $P$. relictum were aligned with the available chitinase gene sequences from Plasmodium spp. (P. gallinaceum CHT1: AF064079; CHT2: AY842482; P. berghei CHT1: AJ305256; P. yoelii CHT1: AB106898; P. knowlesi CHT1: XM002257469; P. vivax CHT1: AB106896; P. falciparum CHT1: AF127445; P. reichenowi CHT1: AY842483) using Geneious translation alignment tool. The quality of the alignment was checked by manual inspection. The combined phylogenetic tree for the two copies was constructed in the programme MEGA 5.2 and using a Maximum Likelihood model. Bootstrap values were used in order to obtain a consensus phylogeny using 200 iterations.

\section{Results}

Both lineages of P. relictum (SGS1 and GRW4) had both gene copies encoding for chitinase. The obtained sequences for the lineage SGS1 and GRW4 were $852 \mathrm{bp}$ and $339 \mathrm{bp}$ for the long copy (GenBank accession number KJ452165, KJ452167) and 845 bp and 393 bp for the short copy 


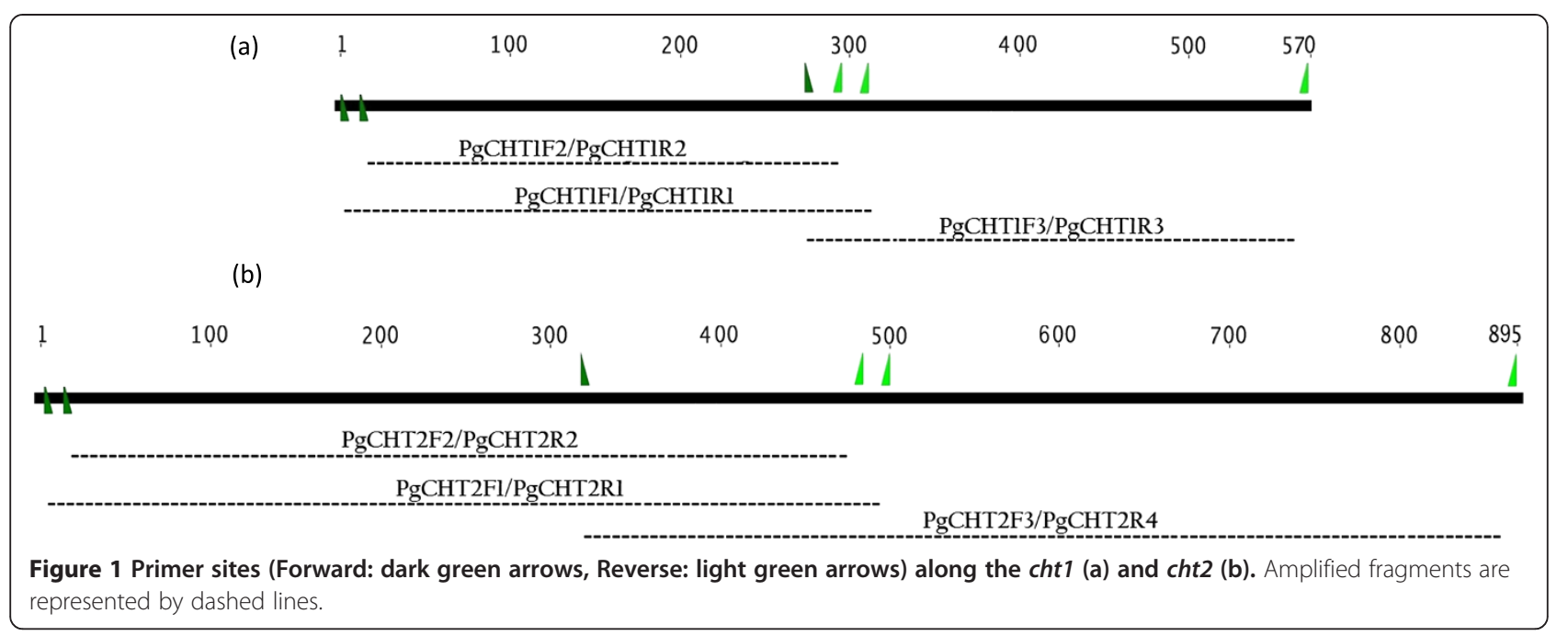

(GenBank accession number KJ452166, KJ452168). Because the obtained sequences from the GRW4 isolate were short only the data from the SGS1 isolate was used for the phylogenetic analyses. These two regions do not completely overlap (Figure 2). As a result, both sequences were trimmed to only cover the shared sites resulting in a combined alignment covering a region of 802 nucleotides. The phylogenetic analyses found strong support for the separation of the long and the short copies, both being present in the SGS1 isolate of P. relictum (Figure 3).

The nucleotide (and amino acid) distances were compared between $P$. gallinaceum and $P$. relictum for both copies using a Pairwise distance matrix. For the long copy a distance of $10.0 \%$ was found (9.2\%) between SGS1 and P. gallinaceum. For the short copy, a distance of $11.0 \%$ (10.6\%) was found between SGS1 and P. gallinaceum. Over the regions for which data of $P$. relictum are available from both isolates of $P$. relictum, SGS1 and GRW4 differed by $1.5 \%(0.8 \%)$ for the short copy and $4.1 \%$ (3.6\%) for the long copy.

\section{Discussion}

The chitinase gene can consist of one or two copies [9], a long and a short one. Previous studies have established that some malaria parasites only have one copy (e.g. $P$. falciparum [10,23] and $P$. berghei [24]) while only $P$. gallinaceum has both variants [9]. Molecular results showed that $P$. relictum has both copies encoding for chitinase (PrCHT1 and PrCHT2). Plasmodium relictum is as far as it is known, the second malaria parasite demonstrated to have both copies. As P. gallinaceum and P. relictum are quite distantly related among the Plasmodium parasites infecting birds $[15,16]$ suggests that the presence of two chitinase gene copies is widespread among the bird

Table 1 Annealing temperature for all the primers used

\begin{tabular}{|c|c|c|c|}
\hline Primer & & Seq (as ordered) & Annealing temp. $\left({ }^{\circ} \mathrm{C}\right)$ \\
\hline PgCHT1_F & Forward & 5'-ATGATAGAAAATCACCAAGACAAATTTTAGA-3' & 50 \\
\hline PgCHT1_R & Reverse & 5'-GGTTCCCAGTCAATATCTACACCA-3' & 50 \\
\hline PgCHT1_F2 & Forward & $5^{\prime}-$ TAGAGGAATACAAAAGAAGGAAACAAGG - $3^{\prime}$ & 50 \\
\hline PgCHT1_R2 & Reverse & 5'-CAGTCAATATCTACACCATCTAAATCA -3' & 50 \\
\hline $\mathrm{PgCHT} 2 \_\mathrm{F}$ & Forward & 5'-ATTCAAGGTTATTATCCATCATGGGT-3' & 53 \\
\hline PgCHT2_R & Reverse & 5'-GAAATCCTATACAGCTCAAAGCTCC - -3' & 53 \\
\hline $\mathrm{PgCHT2} \mathrm{F}_{2}$ & Forward & 5'-GGGTGTCATATAATCATAATATGAAAGA -3' & 53 \\
\hline $\mathrm{PgCHT} 2 \_\mathrm{R} 2$ & Reverse & 5'-GACATTGATATTAATTTATCCTCACACA -3' & 53 \\
\hline PgCHT1_F3 & Forward & 5'-AATGACTTTGATTTAGATGGTGTAGAT-3' & 55 \\
\hline PgCHT1_R3 & Reverse & 5'-TAATTGTTCTTCATAAATAAATGCCA -3' & 55 \\
\hline $\mathrm{PgCHT2}$ _F3 & Forward & 5'-ATGAACCCAATGGATCGTTTGATG -3' & 58 \\
\hline $\mathrm{PgCHT} 2 \_\mathrm{R} 4$ & Reverse & 5'-TAAATTATTAGACAAAGACCACAATCC -3' & 58 \\
\hline
\end{tabular}


Plasmodium parasites. Hence, avian malaria parasites are, to date, the only parasites with both copies. Li et al. [12] suggested that avian malaria parasites could be the ancestor for the chitinase gene in malaria parasites of primates and rodents. Thus, given the current phylogenetic hypothesis, it can be assumed that mammalian parasites evolved from an avian parasite that carried two copies of the chitinase gene.

The bar-coding gene for molecular identification of Plasmodium parasites of birds is the cytochrome $\mathrm{b}$ gene [25-27]. When a genetic difference between lineages exceeds $5 \%$ this is often followed by distinct morphological differentiation which allows for identification of morphological defined species [26]. Obviously, differences are lower when lineages within the same morphological defined species are compared. The MalAvi data base [19] shows that the genetic variability in the cytochrome b between SGS1 and GRW4 is 1.8\% (9 nucleotides different in $480 \mathrm{bp}$ ). However, the present study shows that the genetic variability between SGS1 and GRW4 in the chitinase gene was much higher, $4.1 \%$ (14 different nucleotides in $339 \mathrm{bp}$ ). Moreover, the genetic distance in the cytochrome $\mathrm{b}$ between $P$. relictum and $P$. gallinaceum is $6.9 \%$ (29 nucleotides different in $480 \mathrm{bp}$ ). The results of this study shows that the genetic distance between $P$. relictum and $P$. gallinaceum in the short copy was $13.1 \%$ (44 different nucleotides in 339 bp). Previous studies have identified some nuclear genes with a high variability in $P$. relictum, for instance the msp1 gene [28], that can be used for epidemiological studies of the malaria parasite. In the same way, the chitinase gene could be a good candidate and complement for studies of genetic population structure of the parasites.

In conclusion, the present study demonstrates that the most widespread and harmful avian malaria parasite, $P$. relictum, have the gene encoding for chitinase. In accordance with previous studies on avian malaria (i.e. $P$. gallinaceum), the present study demonstrates that occurrence of both copies (PrCHT1 and PrCHT2) seems to be widespread across avian Plasmodium species. Additionally, the present study demonstrates that the genetic variability of the chitinase gene was high between the two analysed lineages of P. relictum (SGS1 and GRW4).

To determine the phylogenetic relationship between the chitinase gene in malaria parasites, future studies could search for the number of fragments in other species of haemosporidian parasites and most importantly in the genera closely related to Plasmodium that are
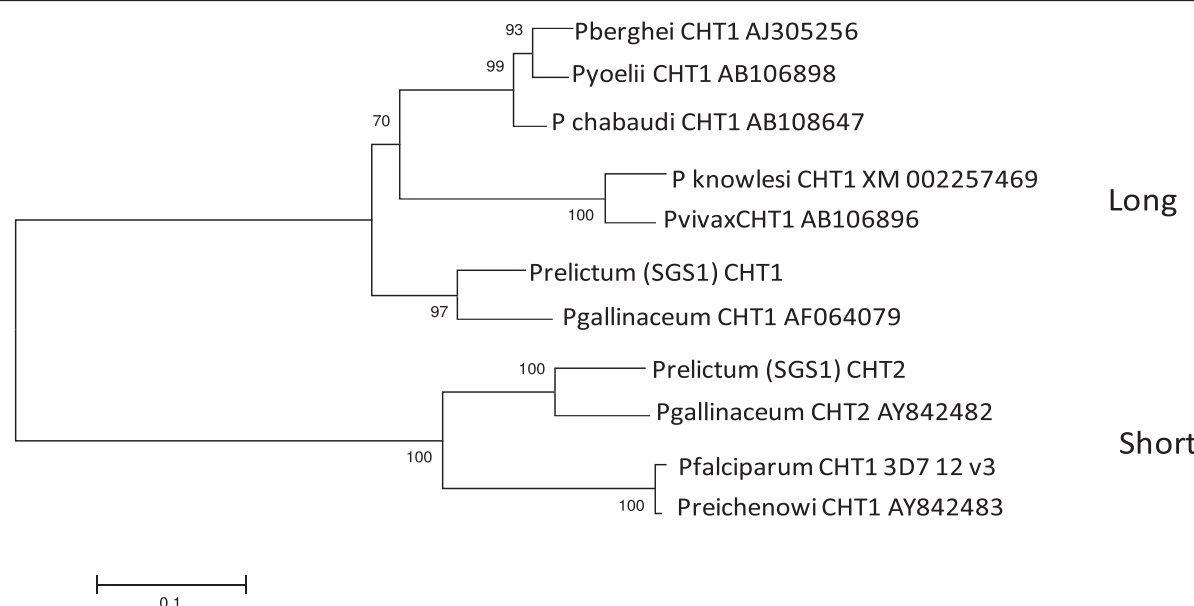

Figure 3 Maximum likelihood consensus phylogeny using midpoint rooting of the translated CHT1 (long copy) gene and CHT2 (short copy) from 10 different malaria species. Numbers in branches represent bootstrap values based on 200 iterations. 
transmitted by vectors of other dipteran families than Culicidae. Another interesting approach would be to analyse the chitinase gene in parasites isolated from a wide range of bird species with a high prevalence of $P$. relictum and different habitat uses, looking at the gene variability in $P$. relictum.

\section{Competing interests}

The authors declare that they have no competing interests.

\section{Authors' contributions}

LG carried out the molecular work on the chitinase gene and drafted the manuscript, $\mathrm{OH}$ provided the samples analysed. SB designed the study and interpreted the data. $\mathrm{OH}$ and SB made important contribution to drafting the manuscript. All authors read and approved the final manuscript.

\section{Acknowledgement}

L. Garcia-Longoria was supported by a PhD grant from Ministry of Economy and Competition of Spain (CGL2009-08976 and CGL2012- 36665). The laboratory work was financially supported by a grant from the Swedish Research Council to S.B. (621-2013-4839).

\section{Author details}

'Departamento de Biología Animal, Universidad de Extremadura, E-06071 Badajoz, Spain. ${ }^{2}$ Department of Biology, Molecular Ecology and Evolution Lab, Ecology Building, Lund University, SE- 22362 Lund, Sweden.

Received: 26 February 2014 Accepted: 7 June 2014

Published: 18 June 2014

\section{References}

1. Sieber KP, Huber M, Kaslow D, Banks SM, Torii M, Aikawa M, Miller LH: The peritrophic membrane as a barrier: its penetration by Plasmodium gallinaceum and the effect of a monoclonal antibody to ookinetes. Exp Parasitol 1991, 72:145-156.

2. Ghosh A, Edwards MJ, Jacobs-Lorena M: The journey of the malaria parasite in the mosquito: hopes for the new century. Parasitol Today 2000, 16:196-201.

3. Perrone JB, Spielman A: Microfilarial perforation of the midgut of a mosquito. J Parasitol 1986, 72:723-727.

4. Schlein Y, Jacobson RL, Messer G: Leishmania infections damage the feeding mechanism of the sandfly vector and implement parasite transmission by bite. Proc Natl Acad Sci U S A 1992, 89:9944-9948.

5. Tsai YL, Hayward RE, Langer RC, Fidock DA, Vinetz JM: Disruption of Plasmodium falciparum chitinase markedly impairs parasite invasion of mosquito midgut. Infect Immun 2001, 69:4048-4054.

6. Li F, Templeton TJ, Popov V, Comer JE, Tsuboi T, Torii M, Vinetz JM: Plasmodium ookinete-secreted proteins secreted through a common micronemal pathway are targets of blocking malaria transmission. J Biol Chem 2004, 279:26635-26644.

7. Kadota K, Ishino T, Matsuyama T, Chinzei Y, Yuda M: Essential role of membrane-attack protein in malarial transmission to mosquito host. Proc Natl Acad Sci U S A 2004, 101:16310-16315.

8. Huber M, Cabib E, Miller LH: Malaria parasite chitinase and penetration of the mosquito peritrophic membrane. Proc Natl Acad Sci U S A 1991, 88:2807-2810.

9. Vinetz JM, Valenzuela JG, Specht CA, Aravind L, Langer RC, Ribeiro JM, Kaslow DC: Chitinases of the avian malaria parasite Plasmodium gallinaceum, a class of enzymes necessary for parasite invasion of the mosquito midgut. J Biol Chem 2000, 275:10331-10341.

10. Vinetz JM, Dave SK, Specht CA, Brameld KA, Xu B, Hayward R, Fidock DA The chitinase PfCHT1 from the human malaria parasite Plasmodium falciparum lacks proenzyme and chitin-binding domains and displays unique substrate preferences. Proc Natl Acad Sci U S A 1999, 96:14061-14066.

11. Boysen KE, Matuschewski K: Arrested oocyst maturation in Plasmodium parasites lacking type II NADH: Ubiquinone Dehydrogenase. J Biol Chem 2011, 286:32661-32671.
12. Li F, Patra KP, Vinetz JM: An anti-chitinase malaria transmission-blocking single-chain antibody as an effector molecule for creating a Plasmodium falciparum-refractory mosquito. J Infect Dis 2005, 192:878-887.

13. Waters AP, Higgins DG, McCutchan TF: Plasmodium falciparum appears to have arisen as a result of lateral transfer between avian and human hosts. Proc Natl Acad Sci U S A 1991, 88:3140-3144.

14. Pick C, Ebersberger I, Spielmann T, Bruchhaus I, Burmester T: Phylogenomic analyses of malaria parasites and evolution of their exported proteins. BMC Evol Biol 2011, 11:167.

15. Perkins SL, Schall JJ: A molecular phylogeny of malarial parasites recovered from cytochrome b gene sequences. J Parasitol 2002, 88:972-978.

16. Martinsen ES, Perkins SL, Schall JJ: A three-genome phylogeny of malaria parasites (Plasmodium and closely related genera): evolution of lifehistory traits and host switches. Mol Phylogenet Evol 2008, 47:261-273.

17. Shahabuddin M, Toyoshima T, Aikawa M, Kaslow DC: Transmissionblocking activity of a chitinase inhibitor and activation of malarial parasite chitinase by mosquito protease. Proc Natl Acad Sci U S A 1993, 90:4266-4270.

18. Valkiūnas G: Avian Malaria Parasites and Other Haemosporidia. Boca Raton: CRC Press; 2005

19. Bensch S, Hellgren O, Pérez-Tris J: MalAvi: a public database of malaria parasites and related haemosporidians in avian hosts based on mitochondrial cytochrome b lineages. Mol Ecol Resour 2009, 9:1353-1358.

20. Palinauskas V, Valkiūnas G, Bolshakov CV, Bensch S: Plasmodium relictum (lineage P-SGS1): effects on experimentally infected passerine birds. Exp Parasitol 2008, 120:372-380

21. Zehtindjiev $P$, llieva M, Westerdahl H, Hansson B, Valkiūnas G, Bensch S: Dynamics of parasitemia of malaria parasites in a naturally and experimentally infected migratory songbird, the great reed warbler Acrocephalus arundinaceus. Exp Parasitol 2008, 119:99-110.

22. Richardson DS, Jury FL, Blaakmeer K, Komdeur J, Burke T: Parentage assignment and extra-group paternity in a cooperative breeder: the Seychelles warbler (Acrocephalus sechellensis). Mol Ecol 2001, 10:2263-2273

23. Langer RC, Vinetz JM: Plasmodium ookinete-secreted chitinase and parasite penetration of the mosquito peritrophic matrix. Trends Parasitol 2001, 17:269-272.

24. Dessens JT, Mendoza J, Claudianos C, Vinetz JM, Khater E, Hassard S, Ranawaka GR, Sinden RE: Knockout of the rodent malaria parasite chitinase pbCHT1 reduces infectivity to mosquitoes. Infect Immun 2001, 69:4041-4047

25. Krizanauskiene A, Hellgren O, Kosarev V, Sokolov L, Bensch S, Valkiūnas G Variation in host specificity between species of avian hemosporidian parasites: evidence from parasite morphology and cytochrome B gene sequences. J Parasitol 2006, 92:1319-1324.

26. Hellgren $\mathrm{O}$, Krizanauskiene A, Valkiünas G, Bensch S: Diversity and phylogeny of mitochondrial cytochrome $B$ lineages from six morphospecies of avian Haemoproteus (Haemosporida: Haemoproteidae). J Parasitol 2007, 93:889-896.

27. Omori S, Sato Y, Isobe T, Yukawa M, Murata K: Complete nucleotide sequences of the mitochondrial genomes of two avian malaria protozoa, Plasmodium gallinaceum and Plasmodium juxtanucleare. Parasitol Res 2007, 100:661-664.

28. Hellgren $\mathrm{O}$, Kutzer $\mathrm{M}$, Bensch $\mathrm{S}$, Valkiūnas $\mathrm{G}$, Palinauskas V: Identification and characterization of the merozoite surface protein 1 (msp1) gene in a host-generalist avian malaria parasite, Plasmodium relictum (lineages SGS1 and GRW4) with the use of blood transcriptome. Malar J 2013, 12:381.

doi:10.1186/1475-2875-13-239

Cite this article as: Garcia-Longoria et al:: Molecular identification of the chitinase genes in Plasmodium relictum. Malaria Journal 2014 13:239. 